\title{
Where should gastric biopsies be performed when areas of intestinal metaplasia are observed?
}

\section{다 (1) $\odot$}

\author{
Authors \\ Institutions \\ 1 Gastroenterology Department, Portuguese Oncology \\ Institute of Porto, Portugal \\ 2 MEDCIDS - Department of Community Medicine, \\ Information and Decision in Health, Faculty of Porto, \\ University of Medicine, Porto, Portugal \\ 3 Surgery and Physiology Department, Faculty of \\ Medicine of the University of Porto, Porto, Portugal
}

Marta Rodríguez-Carrasco, Diogo Libânio ${ }^{1,2}$, Mário Dinis-Ribeiro ${ }^{1,2}$, Pedro Pimentel-Nunes ${ }^{1,2,3}$

\author{
Bibliography \\ DOI https://doi.org/10.1055/a-0953-2247 | \\ Endoscopy International Open 2019; 07: E1636-E1639 \\ (c) Georg Thieme Verlag KG Stuttgart · New York \\ eISSN 2196-9736 \\ Corresponding author \\ Marta Rodríguez-Carrasco, Gastroenterology Department, \\ Portuguese Oncology Institute of Porto, Rua Dr. Bernardino \\ de Almeida, 4200-072 Porto, Portugal \\ Fax: + 351-22-5513646 \\ martarc7@gmail.com
}

Gastric cancer (GC) remains a major health problem worldwide, with a poor 5-year survival rate, partly due to its late diagnosis. Early detection can significantly increase survival and, therefore, identification of patients with higher GC risk (e.g. premalignant conditions) who may benefit from surveillance is essential for decreasing its mortality [1]. Intestinal-type adenocarcinoma, the most frequent histological type, is preceded by a cascade of precancerous lesions such as atrophic gastritis and gastric intestinal metaplasia (IM). Thus, in the absence of screening strategies in European countries, the MAPS II Guideline recommends opportunistic identification and follow-up of individuals at high risk [1], namely those with advanced stages of atrophic gastritis.

Because the correlation between white light endoscopy (WLE) and histological findings is poor and use of chromoendoscopy is cumbersome, two classifications of advanced atrophic changes based on random biopsies have been proposed: Operative Link for Gastritis Assessment (OLGA) and Operative Link on Gastritis/Intestinal Metaplasia (OLGIM). Some reports have shown that IM is the most reliable marker for this purpose due to its higher interobserver agreement, besides the lack of validated endoscopic pattern of atrophic gastritis. Hence, OLGIM to OLGA is preferable, under European Society of Gastrointestinal Endoscopy Guideline MAPS II, for staging gastritis [1]. However, to improve endoscopic diagnostic accuracy, different real-time techniques using imaging-enhanced endoscopy (IEE) emerged. The major advantages are that they are easy to employ and allow precise observation of the entire gastric mucosa and microvascular pattern. Prior reports evaluated the diagnostic efficacy of these technologies, most of them focusing on narrow-band imaging (NBI). A systematic review showed a pooled sensitivity and specificity of NBI of 0.87 and 0.77 , respectively, for IM diagnosis, and 0.90 and 0.83 for dysplasia/cancer diagnosis [2]. More recently, our group reported excellent results in diagnostic yield of NBI in addition to high resolution-WLE (HR-WLE), achieving a global accuracy superior to $90 \%$ in detection of IM and dysplasia [3].

In fact, the concept of random biopsies is now arguable with widespread use of virtual chromoendoscopy (CE) which, at the touch of a button, has been shown to significantly improve endoscopic-histological concordance. Due to the multifocal and patchy distribution presented in most cases of IM, biopsy samples (which represent only a very small part of the entire mucosa) are prone to sampling error, and for these reasons, it seems more logical to rely on endoscopic assessment of the entire mucosa to stratify GC risk instead of depending on random biopsies. But are CE-targeted-biopsies better than SydneyHouston random biopsies for mapping? Results from some reports suggest that targeted biopsies are not inferior, but random biopsies may detect some cases of IM that are not identified with only NBI [4]. However, this difference does not appear to have a significant clinical meaning because most NBI missed areas will be mild and focal IM, so without necessity of surveillance in most cases. In fact, Buxbaum et al analyzed the diagnostic yield of mapping (biopsies according to the updated Sidney protocol) + WLE vs. NBI + WLE vs. NBI + mapping and the best results were with $\mathrm{NBI}+$ mapping (100\% detection of patients with IM and $94.7 \%$ of gastric locations of IM [4]).

Consequently, considering the diagnostic yield of random biopsies and the possibility of missing important changes in 
the mucosa, this approach is being increasingly questioned and the possibility of replacing random with targeted biopsies is being suggested more and more. In this context, a new endoscopic classification of IM (Endoscopic Grading of Gastric Intestinal Metaplasia [EGGIM classification]) was proposed by our group, and recently validated in a multicenter study, showing a sensitivity of $89.4 \%$ for identifying extensive IM (OLGIM III/ IV) if EGGIM > 4 and >99\% accuracy for excluding advance stages of gastritis if EGGIM 0 [5]. In fact, the MAPS II guideline recommends that whenever possible, virtual CE be used to target biopsies instead of performing random biopsies [1]. It would be reasonable to use a combined approach in which WLE would be followed by NBI examination, with exhaustive inspection of the mucosa and EGGIM calculation, in addition to targeted biopsies from suspicious areas for neoplasia; and if no endoscopic suspicion of IM, random biopsies can be performed (only) for Helicobacter pylori $(\mathrm{Hp})$ diagnosis if applicable. This strategy would vary in accordance with different scenarios.

\section{Focal changes with WLE}

Independently of the stage of gastritis, focal gastric lesions may be found with WLE. If some lesions always deserve biopsies (e. g. ulcers) most in fact will be benign changes of the mucosa. In most cases, even though biopsies may still be needed, we recommend always doing CE. With CE, we are able to evaluate mucosal and vascular patterns and endoscopic diagnosis of benignity can be made with more certainty (e.g. fundic glands polyps, papules with foveolar hyperplasia). Therefore, the decision to do biopsies or not depends on the clinical scenario, but commonly at least, fewer biopsies will be needed (while increasing our endoscopic diagnostic confidence).

\section{Normal with WLE, absent IM with virtual CE (EGGIM 0)}

It has been previously reported that in the absence of a typical pattern of IM by NBI, the predictive negative value for extensive $\mathrm{IM}$ is excellent independent of prevalence of IM [3,5]. In this context, we believe that random biopsies to confirm absence of preneoplastic conditions could be spared unless testing for $\mathrm{Hp}$ status is indicated. (in that case, random biopsies according to Sydney System are advised because Hp may be only in the antrum or only in the corpus, although in these cases, a single vial for all the biopsy samples seems adequate). In fact, we have shown that in patients with EGGIM 0 , when sending antrum and corpus biopsies in the same vial, no cases of advancedstage gastritis would be missed without biopsies while only $5 \%$ had OLGA I/II and 2\% OLGIM I/II and in all cases, the pathologist was able to differentiate antrum from corpus mucosa (Castro et al., under revision).

\section{Normal/AG with WLE, focal/moderate IM with virtual CE (EGGIM 1-4)}

When a mucosal pattern B (IM in Pimentel-Nunes classification) is identified but restricted to only one gastric compartment, NBI-targeted-biopsies of that area should be taken in one vial and biopsies from the normal mucosa compartment in a separate vial (so we can confirm the diagnosis and OLGIM stage and also test for $\mathrm{Hp}$ ) ( $\triangleright$ Fig. $\mathbf{1})$.

Nevertheless, we have to acknowledge that these are the cases that we will miss more endoscopically, by underestimating and mostly by overestimating gastritis (for instance, foveolar hyperplasia can overestimate EGGIM because it can be confounded with IM, and patients with severe atrophy without significant IM can be substaged). Thus, relying solely on endoscopic staging in patients with focal/moderate IM is less reliable.

On the other hand, $\mathrm{Hp}$ infection does not seem to affect this endoscopic staging system although $\mathrm{Hp}$ status should be investigated because these patients probably will benefit most from eradication [5]. In our personal experience, NBI-targeted biopsies from IM suspected areas will probably be enough for detection of $\mathrm{Hp}$ status and no additional biopsy from normal area will be needed, because targeted biopsy nearly always includes normal mucosa.

For example, if some focal tubular mucosa is seen only in the antrum/incisura (EGGIM 1-4a), targeted biopsies should be done of these areas (2 to 3 targeted-biopsies in the same vial antrum vial) and then two random biopsies from the corpus (corpus vial) ( $>$ Fig. 2 )

With this approach, individuals who do not benefit from endoscopic surveillance will be identified while at the same time, their Hp status is investigated. However, if additional risk factors are present (e. g. family history of gastric cancer, incomplete IM, autoimmune gastritis, or persistent $\mathrm{Hp}$ infection), surveillance is recommended [1].
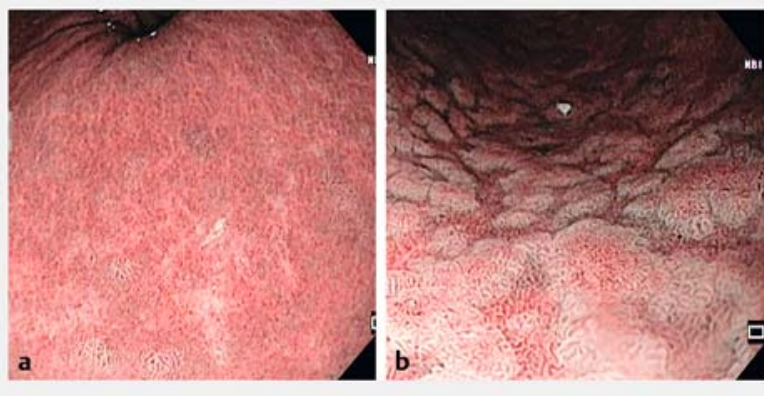

- Fig. $1 \mathrm{NBI}$ examples of EGGIM classification. a A patchy tubular mucosal pattern is identified in the context of atrophic changes. This is a case of focal GIM affecting $<30 \%$ on this area, which corresponds to EGGIM 1 (and probably a global EGGIM 1-4). b Extensive areas with tubular mucosal pattern without irregularity. This is a case of GIM affecting $>30 \%$ of mucosa on this area (therefore, EGGIM 2, and probably global EGGIM $\geq 5$ ). 


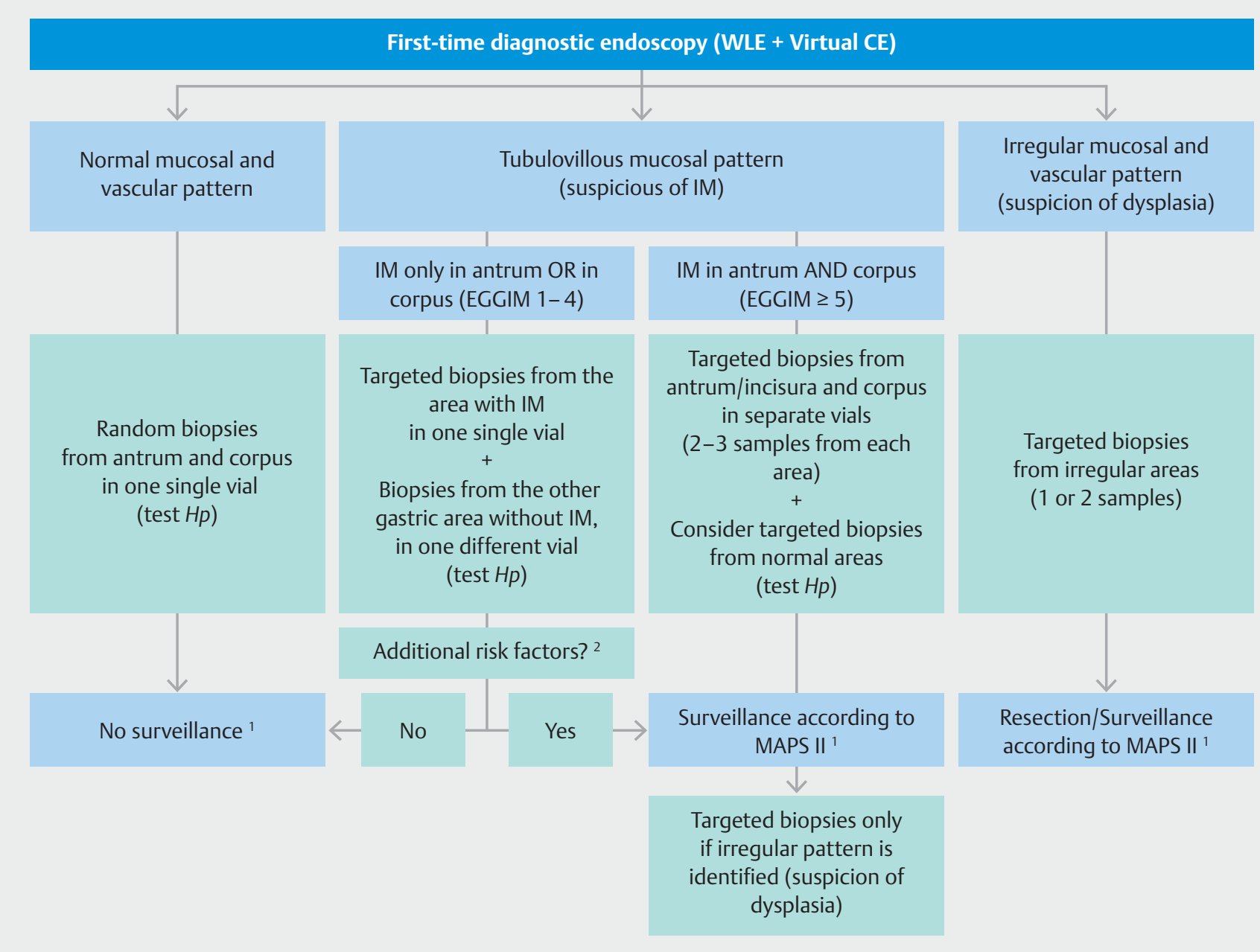

- Fig. 2 Proposed mapping biopsies protocol at initial examination and during follow-up. IM, intestinal metaplasia; EGGIM, endoscopic grading of gastric intestinal metaplasia. ${ }^{1}$ In case of $\mathrm{Hp}$ infection, eradication is recommended. ${ }^{2}$ Family history of gastric cancer, incomplete IM, autoimmune gastritis, or persistent $\mathrm{Hp}$ infection

\section{Extensive IM (EGGIM 5-10)}

When a mucosal pattern B is extensively identified, the question of when and where to do biopsies depends on the specific clinical scenario ( $>$ Fig. 1 ).

For diagnosis (patient not previously known to have IM), NBItargeted biopsies should be taken in separate vials from each location (antrum/incisura and corpus) to identify individuals at high risk. The exception could be in countries with IM prevalence $>25 \%$, where EGGIM positive predictive value for extensive IM is $\geq 85 \%$ and, therefore, in theory biopsies could be avoided [5]. However, in our opinion, in most diagnostic cases, biopsies from the more representative areas in the antrum and in the corpus should be taken in separate vials to also confirm the histological diagnosis. Given the fact that Hp status should be investigated, and that metaplastic glands are frequently not colonized by $\mathrm{Hp}$, taking additional targeted-biopsies from normal areas (e.g. one from the antrum and one from the body) could be considered. These patients do, however, benefit less from $\mathrm{Hp}$ eradication, although some reports suggest that $\mathrm{Hp}$ eradication can halt progression) ( $>$ Fig. 2 ).

However, for surveillance, we believe that biopsies are unnecessary in patients with extensive IM but no irregular/pattern C/ suspicious areas. Even though some authors recommend extensive biopsies in patients under surveillance, in our opinion, most of these biopsies will not alter management. We prefer careful endoscopic evaluation of the entire mucosa, first with WLE and then with virtual CE, and only perform biopsies if pattern $C$ is identified/suspected. If a clear neoplastic lesion is detected, one or two fragments should be taken from the most suspicious areas. If diminutive areas of mucosa with a slightly irregular pattern are seen but with no clearly defined lesion, targeted biopsies of these areas (labelled in separate vials) should be taken. In most cases, these areas will only present IM, but some of them will have focal dysplasia of the epithelium. These patients may benefit from a shorter-interval endoscopic surveillance (in 6 to 12 months, depending if high- or low-grade dysplasia, respectively; [1]). 


\section{Conclusions}

In our opinion, because virtual CE has been proven to optimize the benefit of biopsies, with the proper endoscopic technology, biopsies can be avoided in some circumstances (EGGIM 0 in the initial examination if no $\mathrm{Hp}$ status is needed, potentially if EG$\mathrm{GIM} \geq 5$ in countries with high IM prevalence, and during surveillance of extensive IM). However, biopsy-based protocols are still necessary in other situations, but we recommend changing the approach from random to targeted biopsies whenever possible.

For these reasons, NBI-targeted-biopsies are advised in the following cases:

- To confirm low-risk individuals (EGGIM 1 - 4 without any other risk factors) who will not benefit in most cases from endoscopic surveillance but who will benefit most from $\mathrm{Hp}$ eradication;

- For initial diagnosis of those at high risk (EGGIM $\geq 5$ );

- And, of course, when a dysplastic area is suspected.

It is also important to note that the majority of CE studies were conducted in tertiary centers with former CE experience. Thus, evaluation of diagnostic capability of IEE in community settings is still lacking and IEE needs to be disseminated to better identify patients with high risk of gastric cancer and improve their outcomes. Nevertheless, we believe the focus should be on obtaining a better/high-quality examination with an exhaustive evaluation of mucosal changes and attempt to make a realtime diagnosis, rather than increasing biopsy samples, which sometimes are not representative nor cost-effective. Indubitably, currently we cannot dispense with histological examination, but perhaps in the near future with constant improvement in IEE, it may be possible to restrict biopsies only to confirm dysplastic areas, optimizing performance of our procedures as well as decreasing costs and improving our self-diagnostic yield as endoscopists, which at the same time, could constitute a quality measurement for upper gastrointestinal procedures. In conclusion, not always more biopsies mean better endoscopy!

\section{Competing interests}

None

\section{References}

[1] Pimentel-Nunes P, Libânio D, Marcos-Pinto R et al. Management of epithelial precancerous conditions and lesions in the stomach (MAPS II): European Society of Gastrointestinal Endoscopy (ESGE), European Helicobacter and Microbiota Study Group (EHMSG), European Society of Pathology (ESP), and Sociedade Portuguesa de Endoscopia Digestiva (SPED) guideline update 2019. Endoscopy 2019; 51: 365 - 388

[2] Kikuste I, Marques-Pereira R, Monteiro-Soares M et al. Systematic review of the diagnosis of gastric premalignant conditions and neoplasia with high-resolution endoscopic technologies. Scand J Gastroenterol 2013; 48: $108-1117$

[3] Pimentel-Nunes P, Libanio D, Lage J et al. A multicenter prospective study of the real-time use of narrow-band imaging in the diagnosis of premalignant gastric conditions and lesions. Endoscopy 2016; 48: $723-730$

[4] Buxbaum JL, Hormozdi D, Dinis-Ribeiro M et al. Narrow-band imaging versus white light versus mapping biopsy for gastric intestinal metaplasia: a prospective blinded trial. Gastrointest Endoscopy 2017; 86: $857-865$

[5] Esposito G, Pimentel-Nunes P, Angeletti E et al. Endoscopic grading of gastric intestinal metaplasia (EGGIM): a multicenter validation study. Endoscopy 2019; 51: 515-521 